\title{
Criblage de 4 classes de stupéfiants dans la salive par LC-MS/MS
}

\section{Screening for illicit drugs in oral fluid by $L C-M S / M S$}

Julie SIMONIN, Guillaume SALQUEBRE, Vincent CIRIMELE, Pascal KINTZ*

Laboratoire ChemTox, 3, rue Grüninger, 67400 Illkirch, France

*Auteur à qui adresser la correspondance : Docteur Pascal KINTZ, Laboratoire ChemTox, 3, rue Grüninger, 67400 Illkirch, France - Tél : +33 390400540 - Fax : +33 390400541 - E-mail : pascal.kintz@wanadoo.fr

(Reçu le 28 février 2007 ; accepté après modification le 9 juillet 2007)

\section{RÉSUMÉ}

La sécurité routière est aujourd'hui une des préoccupations majeures des pouvoirs publics. Selon les statistiques et la présence croissante de stupéfiants chez les accidentés de la route de moins de 30 ans, les autorités ont décidé qu'un dépistage rapide de ces substances au bord des routes était nécessaire. Mais deux problèmes se posent. Les techniques déjà mises au point ne permettent pas une détection des 4 classes de stupéfiants à la fois (cannabis, opiacés, cocä̈ne, amphétamines). De plus, les matrices actuellement utilisées ne sont pas adaptées au contrôle routier (prélèvement invasif, atteignant l'intimité de la personne, nécessitant un médecin). C'est pour ces raisons que la salive a été envisagée comme matrice alternative. Le but de ce travail a été de mettre au point une technique qui analyse, dans la salive, les 4 classes de stupéfiants à la fois.

Pour faciliter le recueil de salive, des systèmes de prélèvement ont été développés. Nous avons choisi. d'utiliser le système Intercept ${ }^{\circledR}$, système reconnu par les autorités américaines.

Sur le plan analytique, le dosage par chromatographie liquide parait être la méthode la plus satisfaisante.

Un millilitre $d u$ mélange liquide du système Intercept $($ Salive est extrait par extraction liquide/liquide puis analysé en LC-MS/MS. La séparation se fait sur une colonne XTerra $C_{18}$ avec une élution en mode gradient avec comme solvant : acétonitrile, tampon formiate à $\mathrm{pH}$ 3. Trois

\section{SUMMARY}

Driving under the influence of drugs constitutes an authentic health problem. There are more and more young subjects, less than 30 year old which have road accidents while under the influence of drugs of abuse. Due to sampling difficulties or too large window of detection, conmon specimens such as blood, urine or hair do not fit to the requested situation of driving context. Oral fluid is an alternative biological matrix for drug testing that has several advantages: collection is rapid, non-invasive, and relatively easy to obtain. At this time, there is a lack of a simultaneous screening procedure which is suitable for cannabis, opiates (including heroin), cocaine and amphetamines (including ecstasy). The objective of our work was to develop a single analysis method for the 4 classes of usual drugs.

Analysis by tandem mass spectrometry of this specimen seems to be both simple and highly specific and therefore we have based our study using this approach.

Snall oral fluid samples were collected from volunteers using an Intercept(B) collection device. Samples were prepared by liquid-liquid extraction and analysed by LC-MS/MS. $L C$-separation was achieved using a XTerra MS $C_{\text {is }}$ column, eluted with a gradient of acetonitrile and formate buffer at $p H$ 3. Three intemal standards were used to quantify the drugs: morphine- $d_{3}, M D M A-d_{5}$, and THC- $d_{3}$. The developed method was adequately validated. Good linearity was obtained for all the drugs. Within-day relative coefficients of 
étalons internes sont utilisés pour permettre la quantification des échantillons : morphine- $d_{3}, M D M A-d_{5}$, et THC-d 3 . Les essais de linéarité et répétabilité donnent des résultats satisfaisants. Les coefficients de corrélation varient entre 0,9860 pour le cocaéthylène et 0,9997 pour la cocaüne. Les résultats des tests de répétabilité sont tous inférieurs à $20 \%$, variant de 2,9\% pour la 6-MAM à $19,4 \%$ pour le cocaéthylène. Les limites de quantification, inférieures aux seuils fixés par la SAMSHA, sont de $1 \mathrm{ng} / \mathrm{mL}$ pour toutes les molécules, sauf pour le THC $(2 \mathrm{ng} / \mathrm{mL})$ et l'amphétanine $(5 \mathrm{ng} / \mathrm{mL})$.

Enfin, une série d'échantillons recueillis lors d'un contrôle routier à la sortie d'une discothèque a été analysée par la technique mise au point.

\section{MOTS-CLÉS}

Stupéfiants, salive, système Intercept $(\mathbb{Q}$, contrôle routier.

\section{Introduction}

Ces dernières années, le nombre d'accident de la voie publique, avec présence de stupéfiants dans le sang des conducteurs, est en constante augmentation. Une étude française menée entre 1998 et 1999, par un groupe d'experts de différentes Cours d'Appel, a montré la présence de stupéfiants dans environ $35 \%$ des accidents automobiles corporels et mortels chez les moins de 30 ans (1). Compte tenu de ces chiffres, les pouvoirs publics ont annoncé vouloir dépister le plus rapidement possible les conducteurs sous l'influence de stupéfiants. Mais les matrices actuellement utilisées ne sont pas adaptées aux contraintes du contrôle routier. Cela nécessite une matrice facile à prélever, ne demandant pas la présence d'un médecin (prise de sang), n'atteignant pas l'intimité de la personne lors de son prélèvement (prélèvement d'urine), et où la détection des molécules se fait peu de temps après la prise du stupéfiant et pas dans les mois qui suivent (analyse de cheveux). C'est pour ces raisons qu'une nouvelle matrice a été envisagé : la salive. Elle présente beaucoup d'avantages : prélèvement simple, rapide, non invasif, avec rapidité de détection des molécules après la prise.

Dans la littérature, des études récentes montrent une détection possible du cannabis (2), des opiacés (héroïne (3), morphine (4), codéine (4)), des amphétamines (amphétamine (5), méthamphétamine (5), MDMA (6)), et de la cocaïne (3) dans la salive, mais aussi une cinétique similaire de ces molécules entre plasma et salive (2-6). De plus une corrélation entre présence de stupéfiants dans la salive et signes cliniques, dus à leur absorption, a été démontrée (7).

Pour faciliter le prélèvement de salive et éviter tous les désagréments liés au fait de cracher, des kits de prélèvement ont été mis au point. Celui utilisé pour nos études est le système Intercept(R) (Orasure Technologie, USA), reconnu par la « Food and Drug Administration » (FDA). Il est composé d'un bâtonnet variation (CV) varied from 2,9\% for 6-MAM to $19,4 \%$ for cocaethylene. The limits of quantification were $1 \mathrm{ng} / \mathrm{mL}$ for all drugs, except for $T H C(2 \mathrm{ng} / \mathrm{mL})$ and amphetamine $(5$ $n g / n L L)$.

A series of samples collected of a road control was analyzed by this new method.

\section{KEY-WORDS}

Drugs of abuse, Oral fluid, Intercept@, road control.

avec, à son extrémité, un coton absorbant et d'un tampon de conservation de la salive (liquide bleu).

Peu de techniques d'analyse des stupéfiants dans la salive ont été publiées. Une seule technique, pour l'instant, utilise le système Interceptß comme mode de prélèvement (8). Mais cette dernière ne permet la détection que du THC, molécule active du cannabis. Trois autres techniques d'analyse ont été publiées avec d'autres systèmes de prélèvement : Salivette $(9,10)$, Omni-Sal@ (11). Aucune de ces techniques ne permet un criblage dans la salive des 4 classes de stupéfiants à la fois, avec des seuils de détection inférieurs à ceux fixés par l'autorité américaine SAMSHA (tableau I).

\section{Matériels et méthodes}

\section{Molécules et réactifs}

L'acide formique est acheté chez Prolabo (Fontenay sous Bois; France). Le méthanol, l'heptane, le dichlorométhane et l'isopropanol sont achetés chez Carlo Erba (Val de Reuil, France). Le phosphate d'ammonium, l'acide chlorhydrique et l'acétonitrile sont achetés chez Merck (Darmstadt, Allemagne). Le formiate d'ammonium est acheté chez Sigma-Aldrich

Tableau I : Seuils de détection des stupéfiants dans la salive défnie par l'autorité anéricaine SAMSHA.

\begin{tabular}{|c|c|}
\hline Molécules & Seuil de détection $(\mathbf{n g} / \mathbf{m L})$ \\
\hline Amphétamine & 50 \\
\hline Benzoylecgonine (BZE) & 8 \\
\hline Cocaïne & 8 \\
\hline Codéine & 40 \\
\hline 6-MAM & 4 \\
\hline MDA & 50 \\
\hline MDEA & 50 \\
\hline MDMA & 50 \\
\hline Methamphétamine & 50 \\
\hline Morphine & 40 \\
\hline THC & 2 \\
\hline
\end{tabular}


(Steinheim, Suisse). Tous les solvants sont de qualité HPLC. L'eau ultra-pure est fournie par le distributeur Direct-Q ${ }^{\mathrm{IMS}}$ de Millipore (Molsheim, France).

Les solutions standards de stupéfiants sont fournis par : LGC Promochem (Molsheim, France) pour amphétamine, benzoylegonine, cocaéthylène, cocaïne, codéine, MDA, MDEA, MDMA, et la morphine ; Euromedex (Souffelweyersheim, France) pour 6-MAM, méthamphétamine, MDMA- $\mathrm{d}_{5}$, morphine- $\mathrm{d}_{3}$, THC, et THC- $\mathrm{d}_{3}$. Les molécules deutérées sont reçues à la concentration de $0,1 \mathrm{~g} / \mathrm{L}$, et diluées dans du méthanol. Pour les molécules non deutérées, la concentration est de $1 \mathrm{~g} / \mathrm{L}$, et le solvant de dilution est le méthanol sauf pour la 6-MAM et la cocaüne qui sont diluées dans de l'acétonitrile.

\section{Extraction}

$50 \mu \mathrm{L}$ de THC-d à $1 \mathrm{mg} / \mathrm{L}, 50 \mu \mathrm{L}$ de MDMA-d $\mathrm{d}_{5}$ et de morphine- $\mathrm{d}_{3}$ à $0,1 \mathrm{mg} / \mathrm{L}$, ainsi que $500 \mu \mathrm{L}$ de tampon phosphate à $\mathrm{pH} 8,4$ sont ajoutés à $1 \mathrm{~mL}$ du mélange tampon de conservation du système Intercept@/Salive. L'échantillon est extrait avec $5 \mathrm{~mL}$ du mélange dichlorométhane/isopropanol/n-heptane (25/10/65) par agitation pendant $15 \mathrm{~min}$. Après centrifugation à $3000 \mathrm{rpm}$ et pendant $15 \mathrm{~min}$, la phase organique est récupérée et évaporée sous vide. L'extrait sec est repris avec $70 \mu \mathrm{L}$ de phase mobile aux conditions initiales de l'analyse chromatographique. $10 \mu \mathrm{L}$ sont injectés.

\section{Analyse en LC-MS/MS}

L'analyse chromatographique est réalisée par le système Alliance 2695 (Waters). Les molécules sont séparées grâce à une colonne XTerra $\mathrm{MS} \mathrm{C}_{18}(2,1 \times 100 \mathrm{~mm}$, $3,5 \mu \mathrm{m}$ ) (Waters) avec un gradient composé de 2 solvants : acétonitrile et tampon formiate $\mathrm{pH} 3$ (Tableau II). La colonne est maintenue à $30^{\circ} \mathrm{C}$ pendant la durée de l'analyse. L'acquisition et le traitement des données sont effectués par le logiciel Mass Lynx 4.0 (Waters).

Un Quattro Micro (Micromass) est utilisé pour la détection. L'ionisation est effectuée en mode électrospray positif $($ ESI +$)$. Les conditions optimales d'analyses sont : tension de capillaire, $3,0 \mathrm{kV}$; température de la source ; $120^{\circ} \mathrm{C}$; température du gaz de désolvatation, $500^{\circ} \mathrm{C}$; débit du gaz de désolvatation ; $800 \mathrm{l} / \mathrm{h}$.
Les transitions en MRM (Multiple Reaction Monitoring), les tensions de cône et les énergies de collision sont déterminées par infusion de la molécule dans le spectromètre de masse. Le temps d'enregistrement des transitions a été segmenté en 3 parties en fonction du temps de rétention. Les transitions en MRM avec les segments d'enregistrement correspondant, les tensions de cône et les énergies de collision optimales pour chaque molécule sont reportées dans le tableau III. La pression du gaz de collision est maintenue à $4.10^{-3}$ Bar.

\section{Prélèvement salivaire}

Pour les différents essais, la salive est collectée par le système Intercept ${ }^{\circledR}$ (Orasure Technologies®) USA) selon les instructions données par le fabriquant (www.4intercept.com). Le coton est placé entre joue et gencive pendant environ 2 min, puis introduit dans le tampon de conservation. Le mélange tampon de conservation du système Intercept(B/Salive est utilisé en l'état pour l'extraction.

L'étude sur échantillons réels a été faite sur des salives prélevées lors d'un contrôle routier à la sortie d'une discothèque, tandis que tous les autres échantillons ont été collectés chez des volontaires sains.

\section{Effet matrice}

Ce paramètre permet d'évaluer l'éventuelle suppression ou augmentation de signal due à la matrice de l'échantillon.

Huit échantillons de salive prélevés sur huit personnes différentes ont été dopés après la phase d'extraction avec les différents stupéfiants ( $10 \mathrm{ng} / \mathrm{mL}$ pour le $\mathrm{THC}$ et $100 \mathrm{ng} / \mathrm{mL}$ pour les autres stupéfiants). Ces résultats sont comparés aux résultats obtenus lors de l'analyse par cette méthode des solutions pures.

Les pourcentages sont calculés en prenant comme référence les aires sous la courbe (AUC) des solutions pures. Effet matrice $=100$-(AUC de l'échantillon de salive pour un stupéfiant particulier/AUC de la solution pure de ce même stupéfiant)* 100

Un pourcentage positif indique une suppression de signal, et inversement pour un résultat négatif.

Tableau II : Gradient utilisé pour l'analyse des stupéfiants.

\begin{tabular}{|c|c|c|c|}
\hline Temps (min) & Acétonitrile & Tampon formiate $\mathbf{p H ~ 3}$ & Débit (ml/min) \\
\hline 0 & 5 & 95 & 0,2 \\
\hline 1 & 5 & 95 & 0,2 \\
\hline 8,50 & 80 & 20 & 0,2 \\
\hline 8,60 & 80 & 20 & 0,5 \\
\hline 13 & 80 & 20 & 0,5 \\
\hline 16 & 5 & 95 & 0,2 \\
\hline 26 & 5 & 95 & 0,2 \\
\hline
\end{tabular}


Annales de Toxicologie Analytique, vol. XIX, n² 2, 2007

Tableau III : Tableau récapitulatif des transitions, des tensions de cône, de l'énergie de collision, des temps de rétention et des segments de tous les stupéfiants testés.

\begin{tabular}{|c|c|c|c|c|c|}
\hline Molécules & Transitions & Tension de cône (V) & Énergie de collision $(\mathrm{eV})$ & $\operatorname{Tr}(\min )$ & Segment \\
\hline \multirow[t]{4}{*}{ Morphine } & $286.22>153.06$ & 45 & 39 & \multirow[t]{4}{*}{2,53} & \multirow[t]{4}{*}{1} \\
\hline & $286.22>165.09$ & 45 & 39 & & \\
\hline & $286.22>201.15$ & 45 & 27 & & \\
\hline & $286.22>286.21$ & 45 & 10 & & \\
\hline \multirow[t]{4}{*}{ Morphine $d_{3}$} & $288.95>153.05$ & 46 & 35 & \multirow[t]{4}{*}{2,48} & \multirow[t]{4}{*}{1} \\
\hline & $288.95>165.08$ & 46 & 35 & & \\
\hline & $288.95>181.08$ & 46 & 35 & & \\
\hline & $288.95>289.24$ & 46 & 10 & & \\
\hline \multirow[t]{3}{*}{ Amphétamine } & $135.95>90.91$ & 15 & 17 & \multirow[t]{3}{*}{6,97} & \multirow[t]{3}{*}{2} \\
\hline & $135.95>118.98$ & 15 & 8 & & \\
\hline & $135.95>135.93$ & 15 & 4 & & \\
\hline \multirow[t]{3}{*}{ Méthamphétamine } & $149.90>64.77$ & 18 & 43 & \multirow[t]{3}{*}{7,66} & \multirow[t]{3}{*}{2} \\
\hline & $149.90>90.78$ & 18 & 15 & & \\
\hline & $149.90>118.22$ & 18 & 10 & & \\
\hline \multirow[t]{3}{*}{$\mathrm{MDA}$} & $180.07>104.91$ & 17 & 19 & \multirow[t]{3}{*}{7,66} & \multirow[t]{3}{*}{2} \\
\hline & $180.07>163.08$ & 17 & 11 & & \\
\hline & $180.07>180.22$ & 17 & 4 & & \\
\hline \multirow[t]{3}{*}{ MDMA } & $194.08>104.98$ & 16 & 22 & \multirow[t]{3}{*}{7,91} & \multirow[t]{3}{*}{2} \\
\hline & $194.08>132.99$ & 16 & 20 & & \\
\hline & $194.08>163.08$ & 16 & 12 & & \\
\hline \multirow[t]{3}{*}{ MDMA d $_{5}$} & $199.16>106.93$ & 22 & 25 & \multirow[t]{3}{*}{7,85} & \multirow[t]{3}{*}{2} \\
\hline & $199.16>165.10$ & 22 & 13 & & \\
\hline & $199.16>199.21$ & 22 & 5 & & \\
\hline \multirow[t]{3}{*}{ MDEA } & $208.10>104.91$ & 23 & 23 & \multirow[t]{3}{*}{8,35} & 2 \\
\hline & $208.10>163.14$ & 23 & 13 & & \\
\hline & $208.10>208.24$ & 23 & 6 & & \\
\hline BZE & $290.08>104.98$ & 27 & 33 & 8,48 & 2 \\
\hline & $290.08>168.21$ & 27 & 19 & & \\
\hline & $290.08>290.27$ & 27 & 7 & & \\
\hline Codéine & $300.17>199.14$ & 38 & 31 & 6,82 & 2 \\
\hline & $300.17>300.29$ & 38 & 10 & & \\
\hline Cocaïne & $304.18>182.20$ & 30 & 19 & 8,73 & 2 \\
\hline & $304.18>304.23$ & 30 & 8 & & \\
\hline $\mathrm{CE}$ & $318.25>196.29$ & 31 & 21 & 8,91 & 2 \\
\hline & $318.25>318.30$ & 31 & 6 & & \\
\hline 6-MAM & $328.20>165.19$ & 38 & 42 & 7,88 & 2 \\
\hline & $328.20>211.15$ & 38 & 28 & & \\
\hline & $328.20>328.25$ & 38 & 12 & & \\
\hline THC & $315.15>123.08$ & 30 & 30 & 12,22 & 3 \\
\hline & $315.15>135.17$ & 30 & 22 & & \\
\hline & $315.15>193.17$ & 30 & 22 & & \\
\hline & $315.15>315.29$ & 30 & 8 & & \\
\hline $\mathrm{THC} \mathrm{d}_{3}$ & $318.32>123.00$ & 38 & 21 & 12,23 & 3 \\
\hline & $318.32>123.04$ & 38 & 32 & & \\
\hline & $318.32>196.21$ & 38 & 21 & & \\
\hline & $318.32>318.27$ & 38 & 11 & & \\
\hline
\end{tabular}




\section{Validation de la méthode}

Les droites de linéarité pour tous les stupéfiants ont été obtenues de la même manière : des échantillons de salive, négatif vis-à-vis des stupéfiants, sont dopés avec différentes concentrations (tableau IV).

La répétabilité est obtenue en analysant 7 fois la même salive avec la même concentration en stupéfiants $(10 \mathrm{ng} / \mathrm{mL}$ pour le $\mathrm{THC}$, et $100 \mathrm{ng} / \mathrm{mL}$ pour les autres stupéfiants). Le résultat est donné par le coefficient de variation (CV). Sa valeur doit être inférieure à $20 \%$ pour valider l'essai.

La limite de quantification est définie comme étant la réponse où le signal obtenu est égal à 10 fois le rapport signal/ bruit de fond. Cette limite est évaluée en injectant des concentrations décroissantes de stupéfiants dans la salive jusqu'à l'obtention de 10 fois ce rapport.

\section{Rendement d'extraction (RE)}

Huit salives différentes sont dopées avec différentes concentrations de stupéfiants ( $10 \mathrm{ng} / \mathrm{mL}$ pour le THC et $100 \mathrm{ng} / \mathrm{mL}$ pour les autres stupéfiants), avant extraction et après extraction. L'essai réalisé avec l'addition des stupéfiants après extraction est considéré comme référence. $\mathrm{ED}=(\mathrm{AUC}$ addition avant extraction * 100)/ AUC addition après extraction

\section{Échantillons réels}

Lors d'un contrôle routier à la sortie d'un discothèque, les forces de polices ont collecté des prélèvements de salive avec le système Intercept@. Nous avons analysé 16 de ces échantillons avec la technique mise au point.

\section{Résultats et discussion}

Pour chaque classe de stupéfiants, la salive induit un effet différent (tableau V). Pour les opiacés et les cocaïniques, il y a une suppression d'ions, qui est beaucoup plus importante chez les cocainiques que chez les opiacés. Pour le cannabis, l'effet matrice est négligeable, bien que de faibles variations sont à noter. Pour les amphétamines, l'effet matrice change d'une molécule à l'autre. Pour l'amphétamine et la méthamphétamine, la salive a tendance à augmenter le signal. Tandis que pour la MDEA et la MDA, une diminution du signal, donc une suppression d'ions, est observée.

Une bonne linéarité est observée pour les concentrations allant de 2 à $100 \mathrm{ng} / \mathrm{mL}$ pour le THC, de 20 à 2000 pour les amphétamines, de 5 à 1000 pour les opiacés et de 10 à 2000 pour la cocaïne et ses métabolites (tableau VI). Les coefficients de corrélation sont compris entre 0,986 pour le cocaéthylène et 0,9997 pour la cocaïne.

Tableau IV : Concentrations choisies pour les droites de linéarités.

\begin{tabular}{|c|c|c|c|c|c|c|}
\hline Point de gamme & $\mathbf{1}$ & $\mathbf{2}$ & $\mathbf{3}$ & $\mathbf{4}$ & $\mathbf{5}$ & $\mathbf{6}$ \\
\hline THC & $2 \mathrm{ng} / \mathrm{mL}$ & $5 \mathrm{ng} / \mathrm{mL}$ & $10 \mathrm{ng} / \mathrm{mL}$ & $25 \mathrm{ng} / \mathrm{mL}$ & $50 \mathrm{ng} / \mathrm{mL}$ & $100 \mathrm{ng} / \mathrm{mL}$ \\
\hline Amphétaniiniques & $20 \mathrm{ng} / \mathrm{mL}$ & $40 \mathrm{ng} / \mathrm{mL}$ & $100 \mathrm{ng} / \mathrm{mL}$ & $250 \mathrm{ng} / \mathrm{mL}$ & $1000 \mathrm{ng} / \mathrm{mL}$ & $2000 \mathrm{ng} / \mathrm{mL}$ \\
\hline Cocaïniques & $10 \mathrm{ng} / \mathrm{mL}$ & $25 \mathrm{ng} / \mathrm{mL}$ & $100 \mathrm{ng} / \mathrm{mL}$ & $250 \mathrm{ng} / \mathrm{mL}$ & $1000 \mathrm{ng} / \mathrm{mL}$ & $2000 \mathrm{ng} / \mathrm{mL}$ \\
\hline Opiacés & $5 \mathrm{ng} / \mathrm{mL}$ & $10 \mathrm{ng} / \mathrm{mL}$ & $25 \mathrm{ng} / \mathrm{mL}$ & $100 \mathrm{ng} / \mathrm{mL}$ & $500 \mathrm{ng} / \mathrm{mL}$ & $1000 \mathrm{ng} / \mathrm{mL}$ \\
\hline
\end{tabular}

Tableau $V$ : Résultats de l'effet matrice.

\begin{tabular}{|c|c|c|c|c|c|c|c|c|}
\hline Salive & 1 & 2 & 3 & 4 & 5 & 6 & 7 & 8 \\
\hline Morphine & 10 & 23 & 13 & 15 & 31 & 15 & 10 & 14 \\
\hline Codéine & 33 & 23 & 34 & 40 & 31 & 39 & 44 & 36 \\
\hline 6-MAM & 67 & 54 & 67 & 62 & 60 & 63 & 65 & 64 \\
\hline Cocaïne & 95 & 92 & 94 & 92 & 93 & 94 & 91 & 93 \\
\hline $\mathrm{BZE}$ & 90 & 86 & 90 & 87 & 89 & 91 & 87 & 87 \\
\hline $\mathrm{CE}$ & 70 & 67 & 68 & 74 & 64 & 64 & 76 & 69 \\
\hline Amphétamine & -18 & -24 & -13 & -2 & -13 & 5 & 4 & -8 \\
\hline Méthamphétamine & -6 & -15 & -15 & -9 & -20 & -8 & 0 & -18 \\
\hline MDMA & -4 & -13 & -2 & 12 & -5 & 7 & 10 & 1 \\
\hline MDEA & 50 & 40 & 54 & 53 & 52 & 57 & 56 & 52 \\
\hline MDA & 7 & -5 & 3 & 16 & -10 & -4 & 18 & -8 \\
\hline THC & -3 & 0 & -1 & -3 & -4 & 6 & 2 & 8 \\
\hline
\end{tabular}


Les rendements d'extraction varient entre 15\% (pour la benzoylecgonine) et $86 \%$ (pour la MDMA) (tableau VII). Malgré des résultats qui semblent très dispersés, la plupart des molécules ont des rendements d'extraction supérieurs à $50 \%$. Seul la morphine (43\%), le cocaéthylène $(37 \%)$, et la benzoylecgonine $(15 \%)$ ont des rendements d'extraction inférieurs à 50\%, mais l'intensité de leurs signaux permet une détection satisfaisante.

Les coefficients de variation pour l'essai de répétabilité varient entre $2,9 \%$ (pour la 6-MAM) et $19,4 \%$ (pour la BZE) (tableau VIII).

Les figures 1,2 et 3 représentent les 3 segments d'enregistrement des transitions. Les temps de rétention de chaque molécule sont détaillés dans le tableau III.

Les résultats de l'analyse sur les échantillons réels sont détaillés dans le tableau IX. Sur ces 16 échantillons, 8 sont positifs en amphétamine. Les 8 autres sont négatifs vis-à-vis des 4 classes de stupéfiants.

\section{Conclusion}

Une seule et unique méthode validée permet la détection des 4 classes de stupéfiants (cannabis, opiacés, amphétamine, cocaïne), dans la salive. L'analyse de tous les stupéfiants à la fois permet de diminuer la quantité de prélèvement initiale, et un diagnostic rapide.

Tableau VI : Domaine, équation et coefficient de régression des droites de linéarité, ainsi que les limites de quantification.

\begin{tabular}{|c|c|c|c|c|}
\hline Composés & $\begin{array}{c}\text { Domaine de calibration } \\
(\mathbf{n g} / \mathbf{m L})\end{array}$ & $\begin{array}{c}\text { EOquation } \\
(\mathbf{n g} / \mathbf{m L})\end{array}$ \\
\hline TCH & $2-100$ & $\mathrm{y}=50,292 \mathrm{x}+4,5847$ & 0,988 & 2 \\
\hline Amphétamine & $20-2000$ & $\mathrm{y}=67,816 \mathrm{x}+35,827$ & 0,997 & 20 \\
\hline Méthamphétamine & $20-2000$ & $\mathrm{y}=5,7853 \mathrm{x}-21,311$ & 0,997 & 5 \\
\hline MDMA & $20-2000$ & $\mathrm{y}=0,9431 \mathrm{x}+8,4412$ & 0,9993 & 1 \\
\hline MDA & $20-2000$ & $\mathrm{y}=25,171 \mathrm{x}+0,5965$ & 0,9995 & 5 \\
\hline MDEA & $20-2000$ & $\mathrm{y}=8,9009 \mathrm{x}+28,959$ & 0,9996 & 1 \\
\hline Morphine & $5-1000$ & $\mathrm{y}=22,811 \mathrm{x}-11,493$ & 0,9995 & 1 \\
\hline Codéine & $5-1000$ & $\mathrm{y}=9,882 \mathrm{x}-26,433$ & 0,995 & 1 \\
\hline 6-MAM & $5-1000$ & $\mathrm{y}=109,3 \mathrm{x}-13,641$ & 0,993 & 1 \\
\hline Cocaïne & $10-2000$ & $\mathrm{y}=58,915 \mathrm{x}+6,7645$ & 0,9997 & 1 \\
\hline CE & $10-2000$ & $\mathrm{y}=56,416 \mathrm{x}-114,72$ & 0,986 & 5 \\
\hline BZE & $10-2000$ & $\mathrm{y}=378,4 \mathrm{x}+20,908$ & 0,999 & 1 \\
\hline
\end{tabular}

Tableau VII : Résultats obtenus pour le rendement d'extraction..

\begin{tabular}{|c|c|c|c|c|c|c|c|c|c|c|c|c|}
\hline Salive & 1 & 2 & 3 & 4 & 5 & 6 & 7 & 8 & Moyenne & Ecart-type & Variance & CV \\
\hline Morphine & 51,6 & 42,7 & 45,1 & 40,2 & 38,5 & 39,9 & 45,1 & 39,2 & 43 & 4,397 & 19,33 & 10,3 \\
\hline Codéine & 88,3 & 84,4 & 68,6 & 83,3 & 78,0 & 43,9 & 82,1 & 85,0 & 77 & 14,553 & 211,80 & 19,0 \\
\hline 6-MAM & 58,2 & 41,1 & 45,8 & 47,9 & 58,0 & 58,7 & 53,3 & 53,7 & 52 & 6,511 & 42,39 & 12,5 \\
\hline Cocaine & 78,1 & 40,3 & 70,0 & 76,3 & 50,2 & 51,4 & 47,6 & 65,9 & 60 & 14,323 & 205,15 & 23,9 \\
\hline $\mathrm{BZE}$ & 14,3 & 14,7 & 16,0 & 17,4 & 15,2 & 15,0 & 13,4 & 11,8 & 15 & 1,693 & 2,87 & 11,5 \\
\hline $\mathrm{CE}$ & 28,1 & 27,8 & 31,8 & 33,4 & 46,4 & 38,3 & 46,9 & 46,8 & 37 & 8,336 & 69,48 & 22,3 \\
\hline Amphétamine & 71,2 & 62,8 & 73,0 & 64,4 & 54,2 & 67,7 & 60,5 & 63,5 & 65 & 6,010 & 36,12 & 9,3 \\
\hline Méthamphétamine & 81,6 & 70,9 & 87,1 & 64,0 & 50,3 & 65,5 & 64,2 & 56,3 & 67 & 12,201 & 148,87 & 18,1 \\
\hline MDMA & 94,5 & 86,1 & 100,4 & 86,8 & 72,2 & 84,5 & 75,0 & 85,1 & 86 & 9,197 & 84,59 & 10,7 \\
\hline MOEA & 63,4 & 71,3 & 90,0 & 84,6 & 68,9 & 79,2 & 77,7 & 78,3 & 77 & 8,587 & 73,74 & 11,2 \\
\hline $\mathrm{MDA}$ & 84,5 & 66,8 & 76,7 & 61,9 & 52,5 & 53,1 & 64,3 & 64,2 & 66 & 10,879 & 118,36 & 16,6 \\
\hline THC & 89,6 & 68,3 & 74,5 & 66,6 & 45,4 & 56,1 & 70,6 & 72,0 & 68 & 13,002 & 169,04 & 19,2 \\
\hline
\end{tabular}


Tableau VIII : Résultats obtenus pour la répétabilité exprimée en coefficient de variation (\%).

\begin{tabular}{|c|c|c|c|c|c|c|c|c|c|c|c|}
\hline Essai & $\mathbf{1}$ & $\mathbf{2}$ & $\mathbf{3}$ & $\mathbf{4}$ & $\mathbf{5}$ & $\mathbf{6}$ & $\mathbf{7}$ & Moyenne & Écart-type & Variance & $\mathbf{C V}$ \\
\hline Morphine & 5,3 & 5,5 & 5,2 & 5,4 & 5,6 & 5,6 & 5,8 & 5,48 & 0,20 & 0,04 & 3,57 \\
\hline Codéine & 38,8 & 35,5 & 30,0 & 29,2 & 35,7 & 39,8 & 37,6 & 35,24 & 4,12 & 17,01 & 11,71 \\
\hline 6-MAM & 2,1 & 2,2 & 2,1 & 2,1 & 2,1 & 2,2 & 2,2 & 2,14 & 0,06 & 0,00 & 2,87 \\
\hline Cocaïne & 5,4 & 5,8 & 6,0 & 5,1 & 6,1 & 8,2 & 5,9 & 6,07 & 1,01 & 1,02 & 16,63 \\
\hline BZE & 0,4 & 0,4 & 0,3 & 0,3 & 0,4 & 0,5 & 0,4 & 0,38 & 0,07 & 0,01 & 19,36 \\
\hline CE & 9,7 & 10,7 & 8,8 & 8,9 & 10,8 & 10,8 & 9,2 & 9,85 & 0,90 & 0,82 & 9,18 \\
\hline Amphétamine & 0,9 & 0,9 & 0,7 & 0,7 & 0,9 & 0,9 & 0,9 & 0,85 & 0,10 & 0,01 & 11,50 \\
\hline Méthamphétamine & $\mathbf{1 4 , 5}$ & 14,2 & 12,6 & 13,4 & 16,2 & 15,6 & 16,2 & 14,67 & 1,39 & 1,93 & 9,47 \\
\hline MDMA & 86,4 & 87,7 & 82,9 & 80,8 & 85,3 & 92,4 & 96,4 & 87,41 & 5,41 & 29,27 & 6,19 \\
\hline MDEA & 5,2 & 5,7 & 5,2 & 4,6 & 5,3 & 6,4 & 7,0 & 5,63 & 0,83 & 0,69 & 14,71 \\
\hline MDA & 4,0 & 3,9 & 3,8 & 3,5 & 4,5 & 4,4 & 4,2 & 4,04 & 0,35 & 0,12 & 8,69 \\
\hline THC & 0,19 & 0,16 & 0,15 & 0,15 & 0,16 & 0,16 & 0,15 & 0,16 & 0,01 & 0,0002 & 7,79 \\
\hline
\end{tabular}

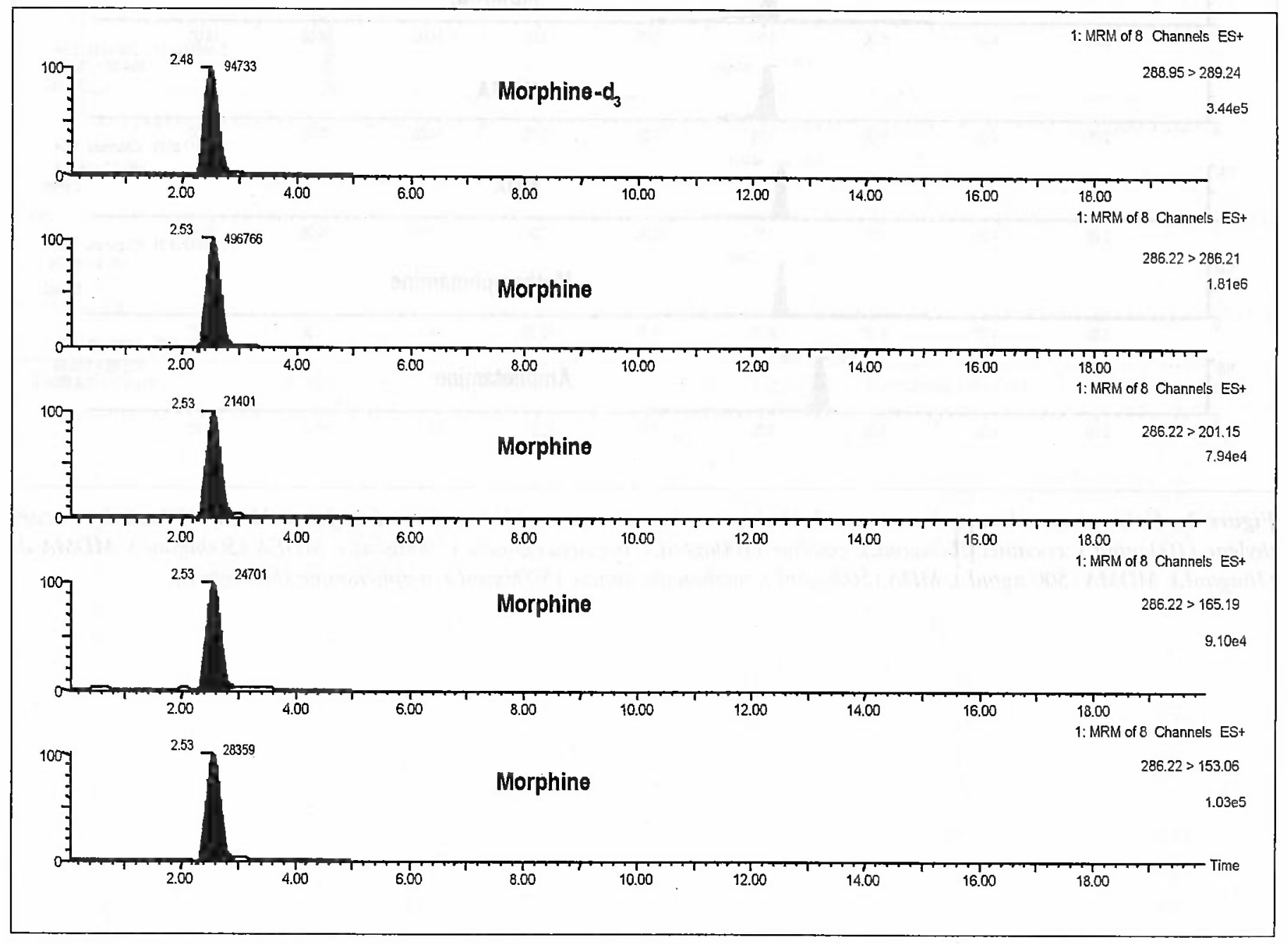

Figure 1 : Calibrateur salivaire du segment 1. De haut en bas morphine-d 3 (1 transition) (16ng/mL), morphine (4 transitions) $(100 \mathrm{ng} / \mathrm{mL})$. 


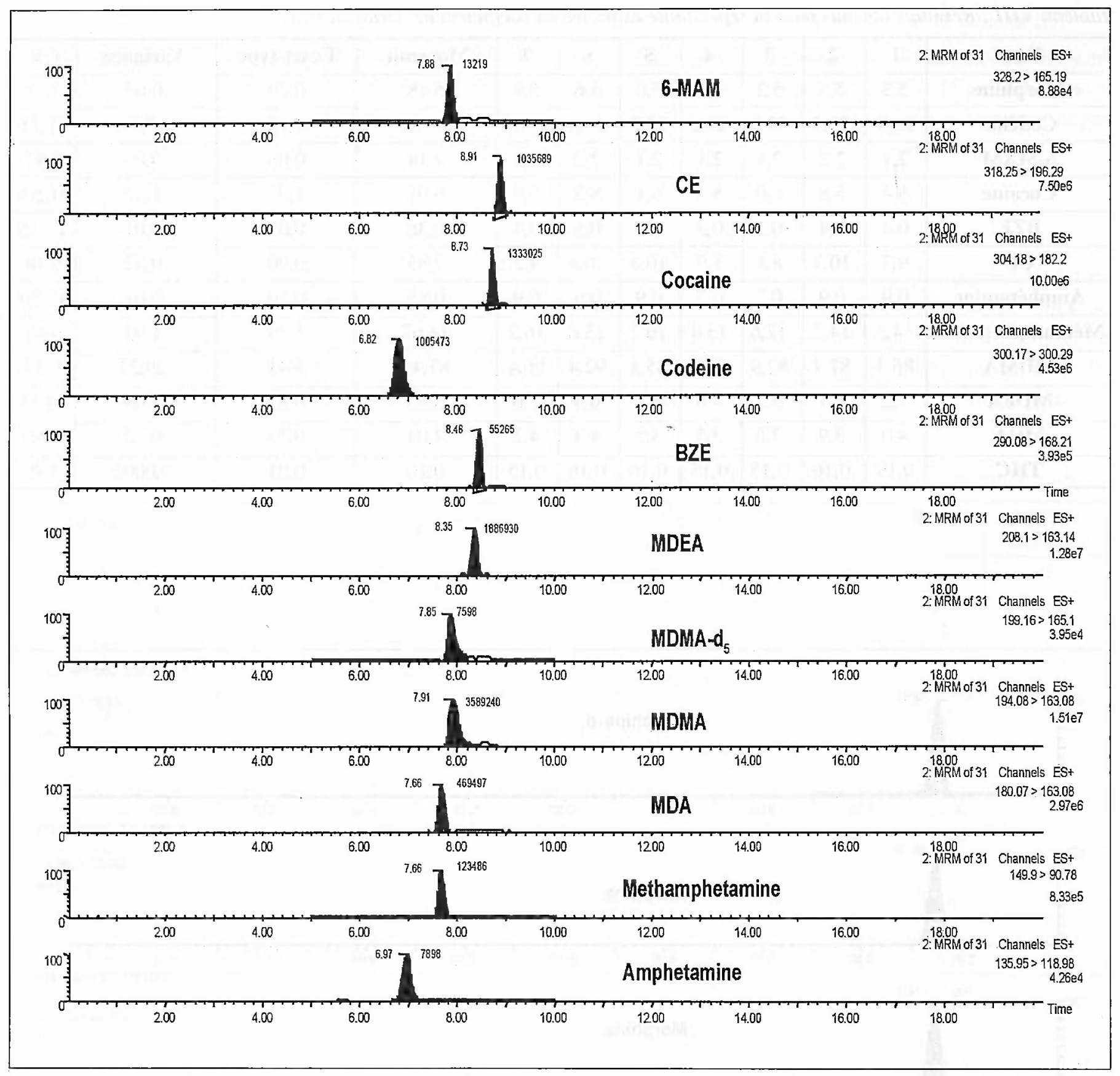

Figure 2 : Calibrateur salivaire du segment 2. De haut en bas avec une seule transition à la fois 6-MAM (100ng/mL), cocaéthylène $(100 \mathrm{ng} / \mathrm{mL})$, cocä̈ne $(100 \mathrm{ng} / \mathrm{mL})$, codéine $(100 \mathrm{ng} / \mathrm{mL})$, benzoylecgonine $(100 \mathrm{ng} / \mathrm{mL})$, MDEA $(500 \mathrm{ng} / \mathrm{mL}), \mathrm{MDMA}-\mathrm{d}_{5}$ (16ng/mL), MDMA (500 ng/mL), MDA (500ng/mL), méthamphétamine (500ng/mL), anpluétamine $(500 \mathrm{ng} / \mathrm{mL})$. 


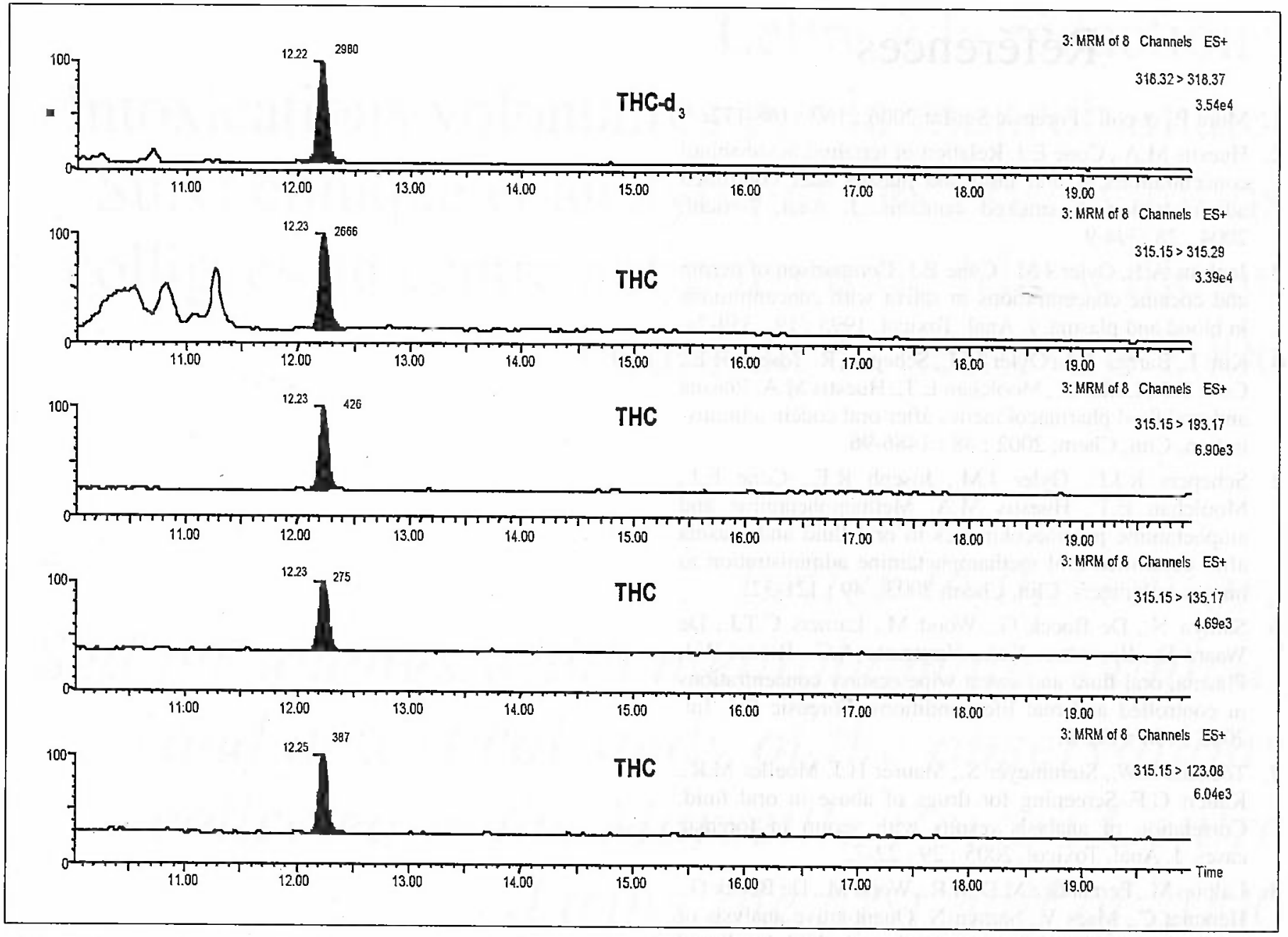

Figure 3 : Calibrateur salivaire du segment 3. De haut en bas $T H C-d_{3}$ (I transition) (I60ng/mL), THC (4 transitions) (50ng/mL).

Tableau IX : Résultats obtenus avec les échantillons prélevés lors d'un contrôle routier à la sortie d'une discothèque (ND = non détecté $; A P=$ amphétamine $; M A P=$ methamphétanine $)$.

\begin{tabular}{|c|c|c|c|c|}
\hline Échantillons & Amphétamines $(\mathbf{n g} / \mathbf{m L})$ & Opiacés $(\mathbf{n g} / \mathbf{m L})$ & Cocaïne $(\mathbf{n g} / \mathbf{m L})$ & THC $(\mathbf{n g} / \mathbf{m L})$ \\
\hline $\mathbf{1}$ & AP 81 & ND & ND & ND \\
\hline $\mathbf{2}$ & AP 182 & ND & ND & ND \\
\hline $\mathbf{3}$ & AP 1121 & ND & ND & ND \\
\hline $\mathbf{4}$ & MAP 2,8 - AP 4,4 & ND & ND & ND \\
\hline $\mathbf{5}$ & MAP 60,2-AP 229-MDMA 10,8-MDA 7,6 & ND & ND & ND \\
\hline $\mathbf{6}$ & AP 223 & ND & ND & ND \\
\hline $\mathbf{7}$ & AP 1,4 - MDMA 21700 & ND & ND & ND \\
\hline $\mathbf{8}$ & MAP 0,22 - AP 391 & ND & ND & ND \\
\hline $\mathbf{9}$ & ND & ND & ND & ND \\
\hline $\mathbf{1 9}$ & ND & ND & ND & ND \\
\hline $\mathbf{1 0}$ & ND & ND & ND & ND \\
\hline $\mathbf{1 1}$ & ND & ND & ND & ND \\
\hline $\mathbf{1 2}$ & ND & ND & ND & ND \\
\hline $\mathbf{1 3}$ & ND & ND & ND & ND \\
\hline $\mathbf{1 4}$ & ND & ND & ND & ND \\
\hline $\mathbf{1 5}$ & ND & &
\end{tabular}




\section{Références}

1. Mura P., et coll., Forensic Sci Int 2006 ; 160 : 168-172.

2. Huestis M.A., Cone E.J. Relation of tetrahydrocannabinol concentrations in oral fluid and plasma after controlled administration of smoked cannabis. J. Anal. Toxicol. 2004 ; $28: 394-9$.

3. Jenkins A.J., Oyler J.M., Cone E.J. Comparison of heroin and cocaine concentrations in saliva with concentrations in blood and plasma. J. Anal. Toxicol. $1995 ; 19: 359-74$.

4. Kim I., Barnes A.J., Oyler J.M., Schepers R., Joseph R.E., Cone E.J., Lafko D., Moolchan E.T., Huestis M.A. Plasma and oral fluid pharmacokinetics after oral codein administration. Clin. Chem; $2002 ; 48: 1486-96$.

5. Schepers R.J.F., Oyler J.M., Joseph R.E., Cone E.J., Moolchan E.T., Huestis M.A. Methamphetamine and amphetamine pharmacokinetics in oral fluid and plasma after controlled oral methamphetamine administration to human volunteers. Clin. Chem. $2003 ; 49$ : 121-32.

6. Samyn N., De Boeck G., Wood M., Lamers C.T.J., De Waard D., Brookhuis K.A., Verstraete A.G., Riedel W.J. Plasma, oral fluid and sweat wipe ecstasy concentrations in controlled and real life conditions. Forensic Sci. Int. $2005 ; 150: 191-8$.

7. Toennes S.W., Steinmeyer S., Maurer H.J, Moeller M.R., Kauert G.F. Screening for drugs of abuse in oral fluid. Correlation of analysis results with serum in forensic cases. J. Anal. Toxicol. $2005 ; 29: 22-7$.

8. Laloup M., Fernandez M.D.M.R., Wood M., De Boeck G., Henquet C., Maes V., Samyn N. Quantitative analysis of tetrahydrocannabinol in preserved oral fluid by liquid chromatography-tandem mass spectrometry. J. Chrom. A. $2005 ; 1082: 15-24$.

9. Teixeira H., Proenca P., Verstraete A., Corte-real F., Nuno Vieira D. Analysis of tetrahydrocannabinol in oral fluid samples using solid-phase extraction and high-performance liquid chromatography-electrospray ionization mass spectrometry. Forensic Sci. Int. 2005 ; 150 : 205-11.

10. Clauwaert K., Decaestecker T., Mortier K., Lambert W., Deforce D., Van Peteghem C., Van Bocxlaer J. The determination of cocaine, benzoylecgonine, and cocaethylene in small-volume oral fluid sample by chromatographyquadrupole-time-of-flight mass spectrometry. J. Anal. Toxicol. $2004 ; 28: 655-9$.

11. Wylie F.M., Torrance H., Anderson R.A., Oliver J.S. Drugs in oral fluid. Part I. Validation of an analytical procedure for licit and illicit drugs in oral fluid. Forensic Sci. Int. $2005 ; 150: 191-8$. 